\title{
On Stokes potential for pressure
}

P.A.Krutitskii

\section{KIAM, dept. 25, Miusskaya Sq.4, Moscow 125047, Russia.}

Potentials for two-dimensional Stokes equations are constructed and studied in [2] in case of closed curves and continuous densities in potentials. In the present paper, we consider the pressure potential specified at the nonclosed arc. In doing so, the densities in the potential belongs to the weighted Hölder space.

By an open arc we mean a simple smooth non-closed arc of finite length without self-intersections [1]. In Cartesian coordinates $x=\left(x_{1}, x_{2}\right) \in \mathbb{R}^{2}$ in a plane we consider a simple smooth open $\operatorname{arc} \Gamma$ parametrized by the arc length $s: \Gamma=\left\{x: x=x(s)=\left(x_{1}(s), x_{2}(s)\right), s \in[a, b]\right\}$. Therefore points $x \in \Gamma$ and values of the parameter $s$ are in one-to-one correspondence. We denote the tangent vector to $\Gamma$ at the point $x(s)$ by $\tau_{s}=(\cos \alpha(s), \sin \alpha(s))$, where $\cos \alpha(s)=x_{1}^{\prime}(s), \sin \alpha(s)=x_{2}^{\prime}(s)$. Let $\mathbf{n}_{s}=(\sin \alpha(s),-\cos \alpha(s))$ be a normal vector to $\Gamma$ at $x(s)$. The direction of $\mathbf{n}_{s}$ is chosen such that it will coincide with the direction of $\tau_{s}$ if $\mathbf{n}_{s}$ is rotated counterclockwise through an angle of $\pi / 2$. The segment $[a, b]$ will be denoted by $\Gamma$ also.

Let the real-valued function $\mu(s)$ of class $\mathbb{C}_{q}^{\omega}[a, b], \omega \in(0,1], q \in[0,1)$ be specified on $\Gamma$. We say that $\mu(s) \in \mathbb{C}_{q}^{\omega}[a, b]$ if $\mu_{0}(s) \in \mathbb{C}^{0, \omega}[a, b]$, where $\mu_{0}(s)=\mu(s)|s-a|^{q}|s-b|^{q}$, and $\mathbb{C}^{0, \omega}[a, b]$ is a Hölder class with the exponent $\omega$.

We consider $\Gamma$ as a cut in a plane. The side of the cut $\Gamma$ which is on the left, when the parameter $s$ increases will be denoted by $\Gamma^{+}$and the opposite side we denote by $\Gamma^{-}$. The goal of the present paper is to study properties of a pressure potential for the Stokes equations [2]:

$$
P\left[\mu_{1}, \mu_{2}\right](x)=P_{c}\left[\mu_{1}\right](x)+P_{s}\left[\mu_{2}\right](x),
$$

where

$$
\begin{gathered}
P_{c}\left[\mu_{1}\right](x)=\frac{1}{2 \pi} \int_{\Gamma} \mu_{1}(\sigma) \frac{\cos (\psi(x, y(\sigma)))}{|x-y(\sigma)|} d \sigma \\
P_{s}\left[\mu_{2}\right](x)=\frac{1}{2 \pi} \int_{\Gamma} \mu_{2}(\sigma) \frac{\sin (\psi(x, y(\sigma)))}{|x-y(\sigma)|} d \sigma \\
\cos \psi(x, y(\sigma))=\frac{x_{1}-y_{1}(\sigma)}{|x-y(\sigma)|}, \quad \sin \psi(x, y(\sigma))=\frac{x_{2}-y_{2}(\sigma)}{|x-y(\sigma)|},
\end{gathered}
$$




$$
y(\sigma)=\left(y_{1}(\sigma), y_{2}(\sigma)\right) \in \Gamma, \quad|x-y(\sigma)|=\sqrt{\left(x_{1}-y_{1}(\sigma)\right)^{2}+\left(x_{2}-y_{2}(\sigma)\right)^{2}} .
$$

Densities $\mu_{1}(s), \mu_{2}(s)$ are assumed to be integrable. Let $z=x_{1}+i x_{2}$, $t=t(\sigma)=\left(y_{1}(\sigma)+i y_{2}(\sigma)\right) \in \Gamma, \quad$ then $\quad d t=t^{\prime}(\sigma) d \sigma=e^{i \alpha(\sigma)} d \sigma$ $z-t(\sigma)=|x-y(\sigma)| e^{i \psi(x, y(\sigma))}$. Assuming that $\mu(s)$ is a real integrable density, we consider the complex potential

$$
\begin{gathered}
P_{E}[\mu](x)=P_{c}[\mu](x)-i P_{s}[\mu](x)= \\
=\frac{1}{2 \pi} \int_{\Gamma} \mu(\sigma) \frac{e^{-i \psi(x, y(\sigma))}}{|x-y(\sigma)|} d \sigma=\frac{1}{2 \pi} \int_{\Gamma} \mu(\sigma) \frac{1}{z-t(\sigma)} d \sigma= \\
=\frac{1}{2 \pi} \int_{\Gamma} \mu(\sigma) e^{-i \alpha(\sigma)} \frac{1}{z-t(\sigma)} d t=-\frac{1}{2 \pi} \int_{\Gamma} \hat{\mu}(t) \frac{1}{t-z} d t
\end{gathered}
$$

where $\hat{\mu}(t)=\mu(\sigma) e^{-i \alpha(\sigma)}$. In $P_{E}[\mu](x)$ we may take either $\mu \equiv \mu_{1}$ or $\mu \equiv \mu_{2}$. Therefore, to study functions $P_{c}\left[\mu_{1}\right](x), P_{s}\left[\mu_{2}\right](x)$, it is sufficient to study a function $P_{E}[\mu](x)$ and to take its real or imaginary part.

We say that the complex-valued function $\hat{\mu}(t)$ belongs to the class $\mathbb{C}_{q}^{\omega}(\Gamma) \quad$ with $\omega \in(0,1], \quad q \in[0,1)$, if $\hat{\mu}_{0}(t) \in \mathbb{C}^{0, \omega}(\Gamma)$, where $\hat{\mu}_{0}(t)=\hat{\mu}(t)|t-t(a)|^{q}|t-t(b)|^{q}$, and $\mathbb{C}^{0, \omega}(\Gamma)$ is a Hölder class with the exponent $\omega$.

Theorem 1. Let $\Gamma$ be an open arc of class $\mathbb{C}^{1, \lambda}, \quad \lambda \in(0,1]$, and $t=t(\sigma)=\left(y_{1}(\sigma)+i y_{2}(\sigma)\right) \in \Gamma$.

1) If $\mu(\sigma) \in \mathbb{C}_{q}^{\omega}[a, b], \omega \in(0,1], q \in[0,1)$, and if $\hat{\mu}(t)=\mu(\sigma) e^{-i \alpha(\sigma)}$, then $\hat{\mu}(t) \in \mathbb{C}_{q}^{\beta}(\Gamma), \beta=\min \{\omega, \lambda\}$.

2) The function $P_{E}[\mu](x)$ belongs to $\mathbb{C}^{0}\left(\overline{\mathbb{R}^{2} \backslash \Gamma} \backslash X\right) \cap \mathbb{C}^{2}\left(\mathbb{R}^{2} \backslash \Gamma\right)$, where $X=x(a) \cup x(b)$ is a set of endpoints of $\Gamma$.

3) The limiting values of the function $P_{E}[\mu](x)$ on $\Gamma^{ \pm}$in interior points are given by the formula

$$
\begin{gathered}
\left.P_{E}[\mu](x)\right|_{x(s) \in \Gamma^{ \pm}}=-i\left( \pm \frac{1}{2} e^{-i \alpha(s)} \mu(s)+\frac{1}{2 \pi i} \int_{\Gamma} \frac{\hat{\mu}(t)}{t-t_{0}} d t\right)= \\
=-i\left( \pm \frac{1}{2} e^{-i \alpha(s)} \mu(s)-\frac{1}{2 \pi i} \int_{\Gamma} \frac{\mu(s) e^{-i \psi(x(s), y(\sigma))}}{|x(s)-y(\sigma)|} d \sigma\right),
\end{gathered}
$$

where $t_{0}=t_{0}(s)=\left(x_{1}(s)+i x_{2}(s)\right) \in \Gamma$ and the integral is understood in a sense of a principle value.

4) If the point $x$ is placed in the neighbourhood of the endpoint $x(d) \quad(d=a$ 
or $d=b)$ and $x \notin \Gamma$, then the estimate holds

$$
\left|P_{E}[\mu](x)\right| \leq \frac{\text { const }}{|x-x(d)|^{\delta}}
$$

where $\delta=q$ if $q \in(0,1)$ and $\delta$ is an arbitrary number from the interval $(0,1)$ if $q=0$.

Remark. By $\mathbb{C}^{0}\left(\overline{\mathbb{R}^{2} \backslash \Gamma} \backslash X\right)$ we denote a class of functions, which are continuous outside $\Gamma$, are continuously extensible on the sides of $\Gamma$ from the left and right in interior points, but there limiting values on $\Gamma$ from the left and right can be different.

Theorem 2. Let $\Gamma$ is an open arc of class $\mathbb{C}^{1, \lambda}, \lambda \in(0,1]$;

$$
\mu(\sigma)=\frac{\eta(\sigma)}{|\sigma-d|^{q}}, \quad q \in(0,1) ; \quad \eta(\sigma) \in \mathbb{C}^{0, \omega}[a, b], \quad \omega \in(0,1] ;
$$

$d=a$ or $d=b$. Let

$$
\hat{\nu}(t)=\hat{\nu}(t(\sigma))=\left(\frac{t(\sigma)-t(d)}{|\sigma-d|}\right)^{q} \eta(\sigma) e^{-i \alpha(\sigma)} .
$$

Then $\hat{\nu}(t) \in \mathbb{C}^{0, \beta}(\Gamma)$, where $\beta=\min \{\omega, \lambda\}$ and for points $z=x_{1}+i x_{2}$, lying near $D=t(d)$ outside $\Gamma$, the asymptotic formula holds

$$
P_{E}[\mu](x)=\mp \frac{e^{ \pm q \pi i}}{2 \sin (\pi q)} \frac{\hat{\nu}(D)}{(z-D)^{q}}+\Theta_{0}(z),
$$

the upper sign is taken if $d=a$, while the lower sign if $d=b$. The function $\Theta_{0}(z)$ is continuous as $z \rightarrow D$ if $\beta>q$. If $0<\beta \leq q<1$, then for $z$, lying near $D=t(d)$ outside $\Gamma$, the estimate holds

$$
\left|\Theta_{0}(z)\right| \leq \frac{\text { const }}{|z-D|^{q_{1}}}
$$

where $q_{1}$ is an arbitrary number, such that $q_{1} \in(q-\beta, q)$. Moreover, $\Theta_{0}(z)$ is analytic in the neighbourhood of $D$ cut along $\Gamma$. By $(z-D)^{q}$ we denote the branch, which is analytic in the neighbourhood of $D$ cut along $\Gamma$ and which takes the value $(t-D)^{q}$ on $\Gamma^{+}$. In addition,

$$
\hat{\nu}(D)=\lim _{\sigma \rightarrow d} \hat{\nu}(t(\sigma)), \quad \text { where } \quad \sigma \in(a, b) .
$$

Theorems 1,2 can be proved by direct verification with the hepl of properties of singular integrals and Cauchy-type integrals from [1]. 
The research was supported by Bernoulli Center at EPFL in Lausanne (Switzerland).

\section{References.}

1. Muskhelishvili N.I. Singular integral equations. Nauka, Moscow, 1968. (In Russian; English translation: Noordhoff, Groningen, 1972.)

2. Popov A.N. Application of potential theory to solving the linearized Navier-Stokes system of equations in a two-dimensional case. Trudy MIAN, 1971, t.116, pp.162-180. (In Russian). 